\title{
A New Approach to Reducing Mortality from Dengue
}

\author{
Sonia Maria Oliveira de Andrade1, Cyntia Maria Moreira Herkert1, \\ Rivaldo Venancio da Cunha ${ }^{1,2^{*}}$, Mariana Delfino Rodrigues' ${ }^{1}$, \\ Baldomero Antonio Kato da Silva ${ }^{3}$ \\ ${ }^{1}$ Post Graduate Program in Infectious and Parasitic Diseases, School of Medicine, Universidade Federal de \\ Mato Grosso do Sul, Campo Grande, Brazil \\ ${ }^{2}$ Fundação Oswaldo Cruz (FIOCRUZ), Campo Grande, Brazil \\ ${ }^{3}$ Physiotherapy Program, Universidade Federal do Piauí, Parnaíba, Brazil \\ Email: soniaufms@gmail.com, cyntiamoreira@yahoo.com.br, ${ }^{*}$ rivaldo venancio@uol.com.br, \\ mdr.enf@gmail.com, ftbaldock@uol.com.br
}

Received 16 December 2013; revised 16 January 2014; accepted 24 January 2014

Copyright (C) 2014 by authors and Scientific Research Publishing Inc.

This work is licensed under the Creative Commons Attribution International License (CC BY).

http://creativecommons.org/licenses/by/4.0/

(c) (i)

Open Access

\section{Abstract}

In 2009, based on a multicenter study conducted in Asia and Latin America and subsidized by the Dengue Control (DENCO) Research Program, the World Health Organization (WHO) proposed a new classification for dengue cases. The purpose of the present study was to evaluate the applicability of the new classification, relative to its previous version [1]. The evaluation, conducted in Campo Grande county, Mato Grosso do Sul state, Brazil, drew on secondary data from referral healthcare centers that assist high-severity dengue patients. A total of 156 medical records of patients with laboratory diagnosis of dengue were investigated. The records covered two epidemic periods: summer of 2006-2007 and summer of 2009-2010. The results showed that $64.6 \%$ of cases classified as dengue fever under the 1997 criteria presented manifestations of severity, warranting their reclassification as dengue with warning signs (49) or severe dengue (15) under the 2009 revised criteria. Bleeding, persistent vomiting, and severe, continuous abdominal pain were the most prevalent warning signs, indicative of risk of progression to severe disease. The revised classification was proved less complex than the current version, facilitating the identification of cases and the clinical management of patients.

\section{Keywords}

Dengue; Disease Classification; World Health Organization

\footnotetext{
${ }^{*}$ Corresponding author.
} 


\section{Introduction}

In Brazil, dengue fever - the arbovirosis most widely distributed among humans — is a disease of mandatory reporting and constitutes a serious public health issue. To date, no vaccine has been developed against the disease [2]. Vector control is deficient, and the spread of infection has remained unchecked. Proper classification of cases and their subsequent management have become a global challenge, since timely, accurate diagnosis is crucial to reducing mortality rates. The current classification of dengue cases, implemented in 1974 and revised in 1997, comprises three levels of severity: dengue fever (DF), dengue hemorrhagic fever (DHF), and dengue shock syndrome (DSS) [1]. Classification of more severe cases is dependent on laboratory results, a practice that has been questioned in recent years, as it can lead to categorizing severe cases such as DF for lack of criteria that take warning signs into account. In 2009, based on a multicenter study conducted in Asia and Latin America, subsidized by the Dengue Control (DENCO) Research Program, the World Health Organization (WHO) proposed a new classification for dengue cases into two categories: dengue fever (which includes cases with and without warning signs) and severe dengue [3]. Warning signs are key to reducing mortality and providing the cases, which are properly classified and managed [4]. The purpose of the present study was to evaluate the applicability of the new classification, relative to its previous version.

\section{Materials and Methods}

The evaluation, conducted in Campo Grande, the capital city of Mato Grosso do Sul state, drew on secondary data culled from referral healthcare centers that assist dengue fever patients. A total of 156 medical records of patients with laboratory diagnosis of dengue were investigated. The records covered two epidemic periods: summer of 2006-2007, with dengue virus type 2 (DENV-2) as the infective agent, and summer of 2009-2010, with DENV-1 and 2.

The study was approved by the Universidade Federal de Mato Grosso do Sul (UFMS) Research Ethics Committee (permit 2174, issued 19 October 2011). Data were collected from November 2011 to January 2012 using a modified version of a WHO form for surveying medical records, originally employed in 18 countries as part of a study on the usefulness and applicability of the WHO revised classification [5].

The form employed in the present study contained nine sections addressing the healthcare center, demography, signs and symptoms, clinical findings, images, laboratory findings, discharge diagnosis as per source document, discharge diagnosis established by reviewer, and laboratory validation.

Discharge diagnosis, established by the attending physician, was based on the 1997 criteria; post-discharge diagnosis by the reviewer was based on the revised 2009 classification [1].

\section{WHO Classifications}

The current OMS classification categorizes the disease as dengue fever (DF), dengue hemorrhagic fever (DHF), or dengue shock syndrome (DSS) [1].

DF is generally benign, self-limited, and rarely fatal, beginning with high fever of sudden onset, accompanied by headache, prostration, myalgia, arthralgia, retro-orbital pain, and maculopapular rash, followed or not by itching. Nausea, vomiting, and diarrhea can occur on the 2nd and 3rd day [1].

DHF is characterized by for simultaneous features: fever (lasting for up to one week); spontaneous bleeding (usually petechiae) or positive tourniquet test; thrombocytopenia $\left(<100,000 \mathrm{~mm}^{3}\right)$; and plasma leakage, characterized by $20 \%$ increase in hematocrit levels or $20 \%$ decrease after the critical stage, or occurrence of pleural effusion, pericardial effusion, or ascites [6].

In DSS, all the defining criteria for DHF may be present in association with circulatory failure, manifested by signs of shock-hypotension; weak, fast pulse rate; convergent blood pressure (differential arterial pressure $<20$ mmHg; cold extremities and cyanosis; prolonged capillary refill time) possibly in conjunction with oliguria and mental confusion [1] [7].

The revised classification (WHO, 2009) considers the following clinical presentations:

Dengue without warning signs: Non-severe cases presenting with the general clinical manifestations of the disease, including fever and pain. These can be concomitant with gastrointestinal manifestations such as nausea, mild vomiting, and diarrhea. Warning signs are absent.

Dengue with warning signs: Cases associated with increased vascular permeability, presenting with abdomin- 
al pain or tenderness; persistent vomiting; fluid accumulation; mucosal bleeding; lethargy or irritability; liver enlargement $(\geq 2 \mathrm{~cm})$; and increase in hematocrit concurrent with rapid decrease in platelet count.

Cases of dengue with warning signs can be further categorized as severe dengue when one or more of the following features are present: hypovolemic shock with plasma leakage, fluid accumulation with respiratory distress, severe bleeding, and severe organ involvement. These cases require emergency treatment or intensive care [4].

\section{Results}

Table 1 shows the classification of cases according to both systems.

The results showed that $64.6 \%$ of cases originally categorized as DF exhibited manifestations of severity, warranting their reclassification as dengue with warning signs (49) or severe disease (15) under the 2009 revised criteria. These patients were treated at lower-complexity healthcare services, but should have been referred to services of greater complexity, given the presence of warning signs, indicative of potentially fatal outcomes.

Table 2 lists the occurrence of clinically relevant warning signs indicative of risk of progression to severe disease in the sample investigated.

Table 1. Reclassification of dengue cases according to the revised WHO (2009) criteria, distributed by discharge diagnosis based on current WHO (1997) categories $(n=156)$.

\begin{tabular}{|c|c|c|c|c|c|c|c|c|}
\hline \multirow{3}{*}{ Current classification } & \multicolumn{6}{|c|}{ Revised classification } & \multicolumn{2}{|c|}{ Total } \\
\hline & \multicolumn{2}{|c|}{ DwoWS } & \multicolumn{2}{|c|}{ DwWS } & \multicolumn{2}{|c|}{ SD } & \multirow[b]{2}{*}{$\mathrm{N}$} & \multirow[b]{2}{*}{$\%$} \\
\hline & $\mathrm{N}$ & $\%$ & $\mathrm{~N}$ & $\%$ & $\mathrm{~N}$ & $\%$ & & \\
\hline $\mathrm{DF}$ & 35 & 22.4 & 49 & 31.4 & 15 & 9.6 & 99 & 63.5 \\
\hline DHF & 5 & 3.20 & 43 & 27.5 & 4 & 2.6 & 52 & 33.3 \\
\hline DwC & 3 & 1.92 & 1 & 0.64 & 1 & 0.64 & 5 & 3.2 \\
\hline Total & 43 & 27.5 & 93 & 59.6 & 20 & 12.8 & 156 & 100 \\
\hline
\end{tabular}

Dengue fever (DF); dengue hemorrhagic fever (DHF); dengue with complications (DwC); dengue without warning signs (DwoWS); dengue with warning signs (DwWS); severe dengue (SD).

Table 2. Comparison of current (WHO, 1997) and revised (WHO, 2009) classifications of dengue cases, considering the presence of warning signs.

\begin{tabular}{|c|c|c|c|c|c|c|c|c|}
\hline \multirow{3}{*}{ Warning signs } & \multicolumn{4}{|c|}{ Current classification } & \multicolumn{4}{|c|}{ Revised classification } \\
\hline & \multicolumn{2}{|c|}{$\begin{array}{c}\mathrm{DF} \\
(n=80)\end{array}$} & \multicolumn{2}{|c|}{$\begin{array}{c}\text { DHF } \\
(n=76)\end{array}$} & \multicolumn{2}{|c|}{$\begin{array}{c}\text { DwWS } \\
(n=93)\end{array}$} & \multicolumn{2}{|c|}{$\begin{array}{c}\text { SD } \\
(n=20)\end{array}$} \\
\hline & $\mathrm{N}$ & $\%$ & $\mathrm{~N}$ & $\%$ & $\mathrm{~N}$ & $\%$ & $\mathrm{~N}$ & $\%$ \\
\hline Bleeding & 21 & 26.2 & 56 & 73.7 & 64 & 68.8 & 13 & 65 \\
\hline Persistent vomiting & 16 & 20 & 23 & 30.3 & 33 & 35.5 & 6 & 30 \\
\hline Severe abdominal pain & 10 & 12.5 & 18 & 23.7 & 24 & 25.8 & 4 & 20 \\
\hline Liver enlargement & 6 & 7.5 & 7 & 9.2 & 12 & 12.9 & 1 & 5 \\
\hline$>$ HCT and $<$ PC & 8 & 10 & 61 & 80.3 & 60 & 64.5 & 9 & 45 \\
\hline Abdominal distension & 1 & 1.2 & 2 & 2.6 & 1 & 1.1 & 2 & 10 \\
\hline Fluid accumulation & 1 & 1.5 & 1 & 1.3 & 3 & 3.2 & 1 & 5 \\
\hline CNS changes & 4 & 5 & 5 & 6.6 & - & - & 2 & 10 \\
\hline
\end{tabular}

Dengue fever (DF); dengue hemorrhagic fever (DHF); dengue without warning signs (DwoWS); Dengue with warning signs (DwWS); severe dengue (SD), increase in hematocrit concurrent with rapid decrease in platelet count; CNS: central nervous system. 
The presence of warning signs in 80 cases originally classified as DF means that only $22.4 \%$ were correctly categorized and properly treated in the ambulatory setting. Misclassification and consequent mismanagement (treatment limited to the primary-care level) were found in $17 \%$ of cases presenting with bleeding, $13 \%$ with persistent vomiting, and $8 \%$ with severe abdominal pain. Potentially lethal aggravation of these cases could be precluded, however, if treatments were based on accurate diagnosis by taking warning signs into account.

The evaluation of diagnostic validity based on disease severity revealed a sensitivity level of 0.4336 , specificity of 0.8139 , positive predictive value of 0.8596 , and negative predictive value of 0.3535 for the current classification, relative to the revised criteria. Reproducibility assessment revealed moderate agreement between current and revised classifications $(\kappa=0.5385, p=0.0021)$.

\section{Discussion}

Because the traditional WHO classification requires four simultaneous criteria to classify cases as DHF, the absence of one or more criteria may lead to misclassification of severe cases-an issue reported by several investigators.

The traditional classification has been challenged on grounds that its complexity can lead to erroneous diagnosis by emphasizing the occurrence of hemorrhage rather than assigning greater weight to plasma leakage [8].

A retrospective study of severe dengue cases conducted in the city of Rio de Janeiro found a trend for belated hospitalization of these patients, combined with too early discharge in some cases, demonstrating that healthcare workers can fail to identify warning signs that should warrant longer hospitalization. Ensuring timely, effective clinical management of dengue patients remains a challenge, despite the need for promptly identifying cases that can progress to severe disease or death [9].

Applying the revised classification to pediatric patients in Indonesia, concluded that this set of criteria can better detect severe cases [10]. Comparing traditional and revised classifications concluded that a high percentage of dengue cases presenting circulatory failure was not correctly identified by current WHO criteria. These findings show that the current categorization is less accurate in identifying severe disease [11].

In a study conducted in 18 countries [5], cases regarded as DF were re-evaluated in the light of the revised classification, revealing a majority of patients presenting warning signs (51.9\%) and some with severe disease (5.7\%) — conditions that require entirely different clinical management, and similar results were found in the present investigation). The study concluded that the revised classification is more sensitive in identifying severe dengue and is simpler to apply in the clinical setting, with a high potential to facilitate clinical management of dengue cases.

Alexander et al. (2011), in a multicenter study conducted in four Latin American and Southeast Asian countries, demonstrated that abdominal pain, mucosal bleeding, and decreased platelet counts are associated with a significantly higher risk of severe illness.

The presence of abdominal pain — a frequent complaint preceding the onset of shock has been unequivocally correlated with increased risk of progression to severe disease [3] [12].

An investigation conducted in India, similar in design to the present study, compared both WHO classifications. The traditional classification was found to categorize the majority of cases (75\%) as DF. One case remained uncategorized. In contrast, the revised system revealed a predominance (82.1\%) of severe dengue cases, in addition to severe manifestations in cases classified as DF. The revised classification was found to be highly sensitive in identifying severe cases, and simple to apply [13]. Based on clinical severity, it takes clinical signs and symptoms as a gold standard, while the current classification is primarily based on laboratory criteria to achieve diagnosis and establish treatment.

Early identification, monitoring of severe forms of the disease, and implementation of appropriate intravenous therapy are measures known to reduce mortality rates [12].

In the present study, the revised WHO (2009) classification was found to be less complex than the set of criteria current employed, facilitating the identification of dengue cases and the clinical management of patients $[1]$.

\section{References}

[1] World Health Organization (1997) Dengue: Guidelines for Diagnosis, Treatment, Prevention and Control. WHO, Geneva. 
[2] Toledo, A.L., Escosteguy, C.C., Medronho, R.A. and de Andrade, F.C. (2006) Confiabilidade do Diagnóstico Final da Dengue Epidemia 2001-2002 no Município do Rio de Janeiro, Brasil. Cadernos de Saúde Pública, 22, 933-940. http://dx.doi.org/10.1590/S0102-311X2006000500006

[3] Alexander, N., Balamseda, A., Coelho, I.C.B., Dimaano, E., Hien, T.T., Hung, N.T., Janisch, T., Kroeger, A., LUM, L.C.S., Martinez, E., Siqueira, J.B., Thuy, T.T., Villalobos, I., Villegas, E. and Wills, B. (2011) Multicentre Prospective Study on Dengue Classification in Tour South-East Asian and Three Latin American Countries. Tropical Medicine and International Health, 16, 936-948. http://dx.doi.org/10.1111/j.1365-3156.2011.02793.x

[4] Organización Panamericana de la Salud (2010) Dengue: Guías de Atención para Enfermos en la Región de las Américas. OPAS, Bolívia.

[5] Barniol, J., Gaczkowski, E.V.B., Cunha, R.V., Salgado, D., Martinez, E., Segarra, C. S., Sandoval, E.B.P., Mishra, A., Laksono, I.S., Lum, L.C., Martinez, J.G., Nunez, A., Balsameda, A., Allende, I., Ramorez, G., Dimaano, E., Thomacheck, K., Akbar, N.A., Ooi, E.E., Villegas, E., Hien, T.T., Farrar, J., Horstick, O., Kroeger, A. and Jaenisch, T. (2011) Usefulness and Applicability of the Revised Dengue Case Classification by Disease: Multicentre Study in 18 Countries. BMC Infectious Diseases, 11, 1-12. http://dx.doi.org/10.1186/1471-2334-11-106

[6] Kalanayanarooj, S., Vaughn, D.W., Nimmannitya, S., Green, S., Suntayakorn, S., Kunentrasail, N., Viramitrachai, W., Ratanavhu-eke, S., Kiatpolpoj, S., Innis, B.L., Rothman, A.L. and Nisalak, A. and Ennis, F.A. (1997) Early Clinical and Laboratory Indicators of Acute Dengue Illness. The Journal of Infectious Diseases, 176, 131-121. http://dx.doi.org/10.1086/514047

[7] Torres, E.M. (2005) A Transmissão. In: Dengue. Fiocruz, Rio de Janeiro.

[8] Horstick, O., Farrar J., Lum, L., Martinez, E., San Martin, J.L., Ehrenberg, J., Velayudhan, R. and Kroeger, A. (2012) Reviewing the Development, Evidence Base, and Application of the Revised Dengue Case Classification. Pathogens and Global Health, 106, 94-101. http://dx.doi.org/10.1179/2047773212Y.0000000017

[9] Gibson, G., Souza-Santos, R., Brasil, P., Pacheco, A.G., Cruz, O.G., Honório O, N.A., Kunelka, C. and Carvalio, M.S. (2013) From Primary Care to Hospitalization: Clinical Warning Signs of Severe Dengue Fever in Children and Adolescentes during na Outbreak in Rio de Janeiro, Brazil. Cadernos de Saúde Pública, 29, 2013.

[10] Basuki, P.S., Budiyanto, Puspitasari, D., Husada, D., Darmowandowo, W., Soegijanto, S. and Yamanaka, A. (2010) Application of Revised Dengue Classification Criteria as a Severity Marker of Dengue Viral Infection in Indonesia. Southeast Asian Journal of Tropical Medicine and Public Health, 41, 1088-1094.

[11] Setiati, T.E., Mairuhu, A., Koraka, P., Supriatna, M., Gillavry, M.R.M., Brandjes, D. P., Osterhaus, A.D., Meer, J.W., Gorp, E.C. and SoemantriI, A. (2007) Dengue Disease Severity in Indonesian Children: An Evaluation of the World Health, Organization Classification System. BMC Infectious Diseases, 7, 1-8. http://dx.doi.org/10.1186/1471-2334-7-22

[12] Singhi, S., Kissoon, N. and Bansal, A. (2007) Dengue and Dengue Hemorrhagic Fever: Management Issues in an Intensive Care Unit. Jornal de Pediatria, 83.

[13] Prasad, D., Kumar, C., Jain, A. and Kumar, R. (2013) Accuracy and Applicability of the Revised WHO Classification (2009) of Dengue in Children Seen at a Tertiary Healthcare Facility in Northern INDIA. Infection, 41, 775-782. http://dx.doi.org/10.1007/s15010-013-0405-3 